\title{
The Impact of the Rotor Slot Number on the Behaviour of the Induction Motor
}

\author{
Konstantinos N. Gyftakis and Joya Kappatou \\ Deptment of Electrical and Computer Engineering, University of Patras, 26500 Patras, Greece \\ Correspondence should be addressed to Konstantinos N. Gyftakis; kosgyftak@upatras.gr
}

Received 10 September 2012; Accepted 13 January 2013

Academic Editor: John Prousalidis

Copyright ( 2013 K. N. Gyftakis and J. Kappatou. This is an open access article distributed under the Creative Commons Attribution License, which permits unrestricted use, distribution, and reproduction in any medium, provided the original work is properly cited.

\begin{abstract}
The impact of the rotor slot number selection on the induction motors is investigated. Firstly, analytical equations will reveal the spatial harmonic index of the air gap magnetic flux density, connected to the geometrical features and the saturation of the induction motor. Then, six motors with different rotor slot numbers are simulated and studied with FEM. The stator is identical in all motors. The motors are examined under time-harmonic analysis at starting and at $1440 \mathrm{rpm}$. Their electromagnetic characteristics, such as electromagnetic torque, stator current, and magnetic flux density, are extracted and compared to each other. The analysis will reveal that the proper rotor slot number selection has a strong impact on the induction motor performance.
\end{abstract}

\section{Introduction}

During the last years, ships have become increasingly dependent on electricity. The new trend to the electric ship design, the integrated electric ship, combines the propulsion system and the ship service electrical system, into a single power system. In this system, the induction motor is used in several applications. Such applications are the propulsion motor, the thrusters, and other various drives, such as pumps and hoists [1].

It is known that the application of electric machines in an electric ship should meet special requirements [2]. Reliability and improved efficiency come first, where there is also a great need for low-noise operation.

In the past, the design and analysis of electric machines were carried out with the use of equivalent circuits and analytical equations. Those methods did offer sufficiently accurate results for a reasonable amount of time. Despite that, those methods did not take into consideration the geometric features of the machine, as well as the complex nonlinear behavior of the magnetic materials.

The rapid advance in computer science, in the last years, gave the opportunity for computational methods to evolve. One of those methods is the finite-element method (FEM).
With the aid of a FEM software, an engineer can design accurately the machine, by also providing the conducting and magnetic properties of the materials used. Then, the simulation carried out solves the Maxwell equations, in space and time, offering a much more accurate solution than the classical methods.

The induction motor design is strongly connected to the power electronics design, since different motor configurations strongly affect its electromagnetic characteristics. This is the reason for extensive research worldwide $[3,4]$.

This paper constitutes a study of the impact of the rotor slot selection on the electromagnetic characteristics of the 4pole, 3-phase, and aluminum cage induction motor, with the use of FEM. In the past, with the aid of analytical calculations and experimental results, many empirical formulas have been proposed concerning the proper selection of the rotor slot number and its influence on cage induction motors [5-7]. The use of FEM offers an insight into the motors' electromagnetic behavior as well as a quantitative representation of their electromagnetic variables and puts the analytical methods under test.

In order to perform a realistic comparison between the designed motors, several aspects had to be taken into consideration. 
Firstly, the stator of all simulated motors should be exactly the same. Secondly, most geometrical rotor variables had to be intact in all motors. So, the rotor shaft diameter, the depth of the rotor bars, and the air gap length are the same in every case. Furthermore, there should be an analogy between the number of the rotor slots and their surface as well as their slot openings. For example, if one doubles the number of rotor slots, he should also have half the slot opening as well as half the bar surface. In this way, the motors have equal rotor resistance and at the same time equal equivalent air gap.

It is obvious that the rotors should be parameterized. For this purpose, a code was written and implemented in the design software. The code was transformed from a previous version [8], which was created in the Laboratory of Electromechanical Energy Conversion, in order to serve this study's requirements.

The stator used for the simulations is chosen from a real motor in the Laboratory, whose resistance was calculated through DC current injection and also whose geometrical variables were known. The used motor is a 3-phase, 4-pole, $4 \mathrm{~kW}$, and $400 \mathrm{~V}$ aluminum cast-cage induction motor.

The simulations carried out are AC time-harmonic and take into account the nonlinear magnetic characteristic of the rotor and stator iron core.

In this work, the authors will firstly present the harmonic index of the radial component of the air gap magnetic flux density with analytical calculations, in a 3-phase induction motor. Then, with the aid of FEM, the results from six simulated induction motors with different rotor slot numbers will be shown and discussed. Five of the motors have even rotor slot numbers, where one has odd. The selected numbers are $24,28,30,40,41$, and 48 . The motors are considered to be unskewed for every case.

\section{Analytical Calculations}

The following calculations will lead to a precise formula of the harmonic content of the magnetic flux density in the induction motor's air gap. The identification of each harmonic's origin is of great value, as it will offer an insight of the air gap magnetic flux density, as well as it will be used later in this work, in order to validate the FEM results.

According to [9], when $q$ is an integer, then the stator's MMF presents space harmonics of rank $v$, where,

$$
v=6 \kappa \pm 1,
$$

and so, the stator MMF can be written as

$$
F_{1}(a, t)=\sum_{\nu=6 \kappa \pm 1}^{\infty} F_{m \nu} \cos (v p \alpha \mp \omega t) .
$$

Also, for the rotor MMF there is

$$
F_{2}(a, t)=\sum_{\mu=1}^{\infty} F_{m \mu} \cos (\mu p \alpha \mp \omega t-\varphi),
$$

where

$$
\mu=\kappa \frac{s_{2}}{p} \pm 1,
$$

and $\alpha$ is the geometrical angle:

$$
a=\frac{1}{p} \frac{\pi}{\tau} x
$$
by

According to [10], the air gap relative permeance is given

$$
\Lambda_{g}(\alpha)=\Lambda_{g 0} \lambda_{g 1}(\alpha) \lambda_{g 2}(\alpha),
$$

where

$$
\begin{gathered}
\Lambda_{g 0}=\frac{\mu_{0}}{k_{c} g}=\frac{\mu_{0}}{g^{\prime}}, \\
\lambda_{g 1}(\alpha)=1+\sum_{k=1,2,3}^{\infty} A_{k} \cos \left(k s_{1} \alpha\right), \\
\lambda_{g 2}(\alpha)=1+\sum_{l=1,2,3}^{\infty} A_{l} \cos \left[l s_{2}\left(\alpha-\omega_{2} t\right)\right], \\
\omega_{2}=2 \pi(1-s) f=2 \pi(1-s) n_{s} p=2 \pi n_{m} p=p \Omega_{m} .
\end{gathered}
$$

$A_{k}$ and $A_{l}$ are related to the stator and rotor slot geometrical variables. Two analytical formulas have been proposed to describe them. The first one is by Dabrowski [11] and Voldek [12] and the second by Heller and Hamata [13] and Weber [14].

So if we introduce (7), (8), and (9) into (6), then

$$
\Lambda_{g}(\alpha, t)=\Lambda_{g 0} \lambda_{g}(\alpha, t)=\frac{\mu_{0}}{k_{C} g} \lambda_{g}(\alpha, t),
$$

where

$$
\begin{aligned}
\lambda_{g}(\alpha, t) & \\
= & 1+\sum_{k=1,2,3}^{\infty} A_{k} \cos \left(k s_{1} \alpha\right)+\sum_{l=1,2,3}^{\infty} A_{l} \cos \left[l s_{2}\left(\alpha-\omega_{2} t\right)\right] \\
& +\frac{1}{2} \sum_{k=1,2,3}^{\infty} \sum_{l=1,2,3}^{\infty} A_{k} A_{l}\left\{\begin{array}{c}
\cos \left[\left(l s_{2}+k s_{1}\right) \alpha-l s_{2} \omega_{2} t\right] \\
+\cos \left[\left(l s_{2}-k s_{1}\right) \alpha-l s_{2} \omega_{2} t\right]
\end{array}\right\} .
\end{aligned}
$$

The first term of (12) describes the equivalent uniform air gap's permeance, the second and third terms describe the harmonics of the stator and rotor permeance, respectively, and the last term represents the harmonics of the permeance due to the reciprocal effect of the stator and rotor.

The radial component of the air gap magnetic flux density can be calculated by

$$
b(\alpha, t)=\left[F_{1}(\alpha, t)+F_{2}(\alpha, t)\right] \Lambda_{g}(\alpha, t)
$$

or if $b(\alpha, t)$ is divided into the stator and rotor MMF contribution to the magnetic flux density:

$$
b(\alpha, t)=b_{1}(\alpha, t)+b_{2}(\alpha, t),
$$

where

$$
\begin{aligned}
& b_{1}(\alpha, t)=F_{1}(\alpha, t) \Lambda_{g}(\alpha, t), \\
& b_{2}(\alpha, t)=F_{2}(\alpha, t) \Lambda_{g}(\alpha, t) .
\end{aligned}
$$


In order to examine each term of $(12), b_{1}(\alpha, t)$ is divided into four components:

$$
b_{1}(\alpha, t)=b_{1}^{1}(\alpha, t)+b_{1}^{2}(\alpha, t)+b_{1}^{3}(\alpha, t)+b_{1}^{4}(\alpha, t),
$$

and each individual term of (16) is

$$
\begin{gathered}
b_{1}^{1}(\alpha, t)=\frac{\mu_{0}}{k_{C} g}\left[\sum_{v=6 \kappa \pm 1}^{\infty} F_{m v} \cos (v p \alpha \mp \omega t)\right], \\
b_{1}^{2}(\alpha, t)=\frac{1}{2} \frac{\mu_{0}}{k_{C} g} \sum_{v=6 \kappa \pm 1}^{\infty} \sum_{k=1,2,3}^{\infty} F_{m v} A_{k} \\
\times\left\{\begin{array}{c}
\cos \left[\left(v p+k s_{1}\right) \alpha \mp \omega t\right] \\
+\cos \left[\left(v p-k s_{1}\right) \alpha \mp \omega t\right]
\end{array}\right\}, \\
b_{1}^{3}=\frac{1}{2} \frac{\mu_{0}}{k_{C} g} \sum_{v=6 \kappa \pm 1}^{\infty} \sum_{l=1,2,3}^{\infty} F_{m v} A_{l} \\
b_{1}^{4}=\frac{1}{4} \frac{\mu_{0}}{k_{C} g} \sum_{v=6 \kappa \pm 1}^{\infty} \sum_{k=1,2,3}^{\infty} \sum_{l=1,2,3}^{\infty} F_{m v} A_{k} A_{l} \\
+\cos \left[\left(v p+l s_{2}\right) \alpha-\left(l s_{2} \omega_{2} \pm \omega\right) t\right] \\
\times\left\{\begin{array}{l}
\cos \left[\left(v p+l s_{2}+k s_{1}\right) \alpha-\left(l s_{2} \omega_{2} \pm \omega\right) t\right] \\
+\cos \left[\left(v p-l s_{2}-k s_{1}\right) \alpha+\left(l s_{2} \omega_{2} \mp \omega\right) t\right] \\
+\cos \left[\left(v p+l s_{2}-k s_{1}\right) \alpha-\left(l s_{2} \omega_{2} \pm \omega\right) t\right] \\
+\cos \left[\left(v p-l s_{2}+k s_{1}\right) \alpha+\left(l s_{2} \omega_{2} \mp \omega\right) t\right]
\end{array}\right\} .
\end{gathered}
$$

The same is for the rotor MMF contribution to the magnetic flux density term:

$$
b_{2}(\alpha, t)=b_{2}^{1}(\alpha, t)+b_{2}^{2}(\alpha, t)+b_{2}^{3}(\alpha, t)+b_{2}^{4}(\alpha, t),
$$

and each individual term of (18) is

$$
\begin{aligned}
& b_{2}^{1}(\alpha, t)=\frac{\mu_{0}}{k_{C} g}\left[\sum_{\mu=1}^{\infty} F_{m \mu} \cos (\mu p \alpha \mp \omega t-\varphi)\right], \\
& b_{2}^{2}= \frac{1}{2} \frac{\mu_{0}}{k_{C} g} \sum_{\mu=1}^{\infty} \sum_{k=1,2,3}^{\infty} F_{m \mu} A_{k} \\
& \times\left\{\begin{array}{c}
\cos \left[\left(\mu p+k s_{1}\right) \alpha \mp \omega t-\varphi\right] \\
+\cos \left[\left(\mu p-k s_{1}\right) \alpha \mp \omega t-\varphi\right]
\end{array}\right\}, \\
& b_{2}^{3}=\frac{1}{2} \frac{\mu_{0}}{k_{C} g} \sum_{\mu=1}^{\infty} \sum_{l=1,2,3}^{\infty} F_{m \mu} A_{l} \\
& \quad \times\left\{\begin{array}{c}
\cos \left[\left(\mu p+l s_{2}\right) \alpha-\left(l s_{2} \omega_{2} \pm \omega\right) t-\varphi\right] \\
+\cos \left[\left(\mu p-l s_{2}\right) \alpha+\left(l s_{2} \omega_{2} \mp \omega\right) t-\varphi\right]
\end{array}\right\},
\end{aligned}
$$

$$
\begin{aligned}
b_{2}^{4}= & \frac{1}{4} \frac{\mu_{0}}{k_{C} g} \sum_{\mu=1}^{\infty} \sum_{k=1,2,3}^{\infty} \sum_{l=1,2,3}^{\infty} F_{m \mu} A_{k} A_{l} \\
& \times\left\{\begin{array}{c}
\cos \left[\mu p \alpha \mp \omega t-\varphi+\left(l s_{2}+k s_{1}\right) \alpha-l s_{2} \omega_{2} t\right] \\
+\cos \left[\mu p \alpha \mp \omega t-\varphi-\left(l s_{2}+k s_{1}\right) \alpha+l s_{2} \omega_{2} t\right] \\
+\cos \left[\mu p \alpha \mp \omega t-\varphi+\left(l s_{2}-k s_{1}\right) \alpha-l s_{2} \omega_{2} t\right] \\
+\cos \left[\mu p \alpha \mp \omega t-\varphi+\left(l s_{2}-k s_{1}\right) \alpha+l s_{2} \omega_{2} t\right]
\end{array}\right\} .
\end{aligned}
$$

From (17) and (19), the space harmonics which depend on the stator and rotor MMF and the rotor and stator slot numbers $s_{1}, s_{2}$, are presented, respectively,

$$
\left\{\begin{array}{c}
v, \\
v \pm \frac{k s_{1}}{p}, \\
v \pm \frac{l s_{2}}{p}, \\
v \pm \frac{\left(l s_{2} \pm k s_{1}\right)}{p}, \\
\mu, \\
\mu \pm \frac{k s_{1}}{p}, \\
\mu \pm \frac{l s_{2}}{p}, \\
\mu \pm \frac{\left(l s_{2} \pm k s_{1}\right)}{p}
\end{array}\right\} \quad \forall v=6 \kappa \pm 1, \mu=\kappa \frac{s_{2}}{p} \pm 1, \kappa, k, l \in \mathbb{N} \text {. }
$$

The equations shown in (20) offer useful information about the magnetic field's distribution. The motors that will be simulated have 4 magnetic poles and 36 stator slots. As a consequence, $p=2$ and $s_{1}=36$, which is a multiple of 3 and 4. So, if the number of rotor slots is also a multiple of 4 , there will be only odd harmonic rank numbers in the magnetic flux density. If the number of rotor slots is a multiple of 2 (e.g., 30 ), then the magnetic flux density would be characterized by both even and odd harmonic rank numbers.

According to $[15,16]$, the air gap relative permeance takes the following form in order to take into account the iron core saturation, considering the fundamental harmonic:

$$
\Lambda(\alpha) \approx \Lambda_{0}+\Lambda_{\text {sat }}(\alpha, t),
$$

where

$$
\Lambda_{\text {sat }}(\alpha, t)=-\Lambda_{\text {sat }} \cos \left(2 p \alpha-2 \omega_{1} t-2 \varphi_{s}\right) .
$$

So, the influence of the rotor and stator MMF on the magnetic flux density due to the saturation will be

$$
b_{\text {sat }}(\alpha, t)=\left[F_{1}(\alpha, t)+F_{2}(\alpha, t)\right] \Lambda_{\text {sat }}(\alpha, t) .
$$


Equation (23) due to (22) results in

$$
\begin{aligned}
b_{\text {sat }}(\alpha, t) & \\
& =-\Lambda_{\text {sat }} \frac{1}{2} \\
& \times\left[\begin{array}{c}
\sum_{v=6 \kappa \pm 1}^{\infty} F_{m v}\left\{\begin{array}{c}
\cos \left[(\nu+2) p \alpha-\left(2 \omega_{1} \pm \omega\right) t-2 \varphi_{s}\right] \\
+\cos \left[(\nu-2) p \alpha+\left(2 \omega_{1} \mp \omega\right) t+2 \varphi_{s}\right]
\end{array}\right\} \\
+\sum_{\mu=1}^{\infty} F_{m \mu}\left\{\begin{array}{c}
\cos \left[(\mu+2) p \alpha-\left(2 \omega_{1} \pm \omega\right) t-\left(\varphi+2 \varphi_{s}\right)\right] \\
+\cos \left[(\mu-2) p \alpha+\left(2 \omega_{1} \mp \omega\right) t-\left(\varphi-2 \varphi_{s}\right)\right]
\end{array}\right\}
\end{array}\right] .
\end{aligned}
$$

From (24), one may observe that the harmonic ranks of the air gap magnetic flux density due to saturation obey to

$$
\left\{\begin{array}{c}
(v \pm 2), \\
(\mu \pm 2)
\end{array}\right\} \quad \forall v=6 \kappa \pm 1, \mu=14 k \pm 1, \kappa \in \mathbb{N}
$$

From (20) and (25), we get that harmonic numbers such as 3,5, and 9 are produced from both the stator slots existence and the iron core magnetic saturation. Furthermore, the analytical solution leads to the result that the space harmonic rank of the air gap magnetic flux density, because of saturation, is always an odd number, as expected.

\section{Creating the Models}

The six simulated motors have the same stator, whose geometrical and electromagnetic variables are known as it is a real stator. The simulated stator is presented in Figure 1. It has 36 stator slots and the 3 phases are delta connected. The stator, for all cases, is fed by symmetrical 3-phase sinusoidal voltage $380 \mathrm{~V}$.

The rotors are created using a parameterized code developed in the Laboratory. The selected rotor slot numbers are $24,28,30,40,41$, and 48 . The shape of the bars is chosen to belong to NEMA's class A design specification. The numbers are selected after a research with analytical equations and previously published works [17]. The created rotors are presented in Figure 2. One can see that the rotor shaft is intact for all cases along with the bars' depth. Also, the slot opening and the surface of the bars are inversely proportional to the rotor slot number. As a consequence, the different motors have equal rotor resistance and equivalent air gap.

For the appropriate meshing of the models, the skin and proximity effects are taken into consideration. The mesh for the motor with 28 rotor slots is presented in Figure 3. The mesh distribution is similar to that of the other models. The numbers of nodes and elements are about 81000 and 161000, respectively, in all models.

\section{The Starting Behavior}

The simulated motors are studied in this paragraph at their starting. The magnetic flux lines distribution is presented in Figure 4. One may observe that the magnetic flux lines do not

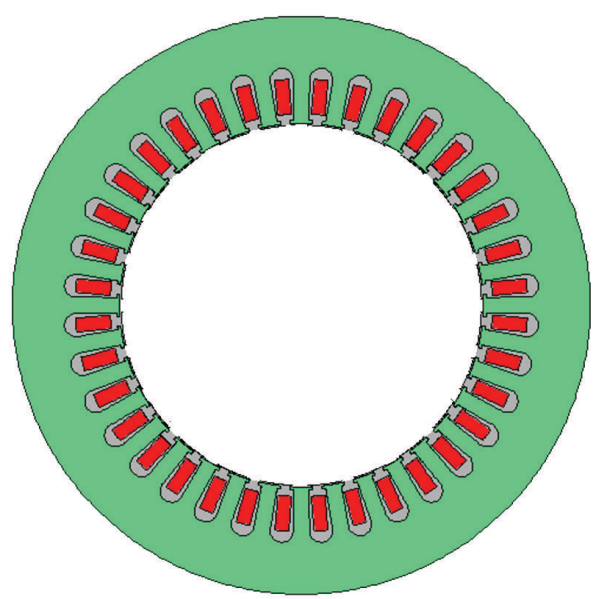

FIgURE 1: The simulated stator.

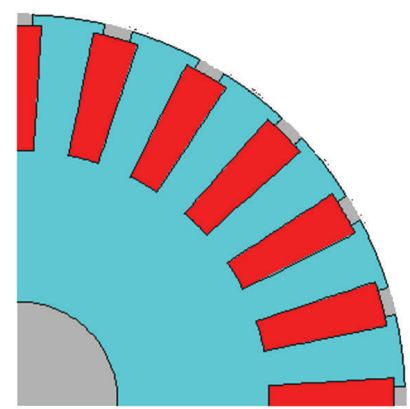

(a)

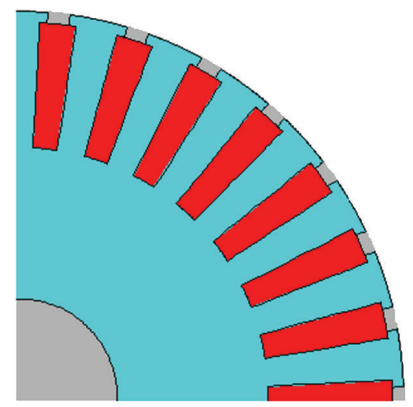

(c)

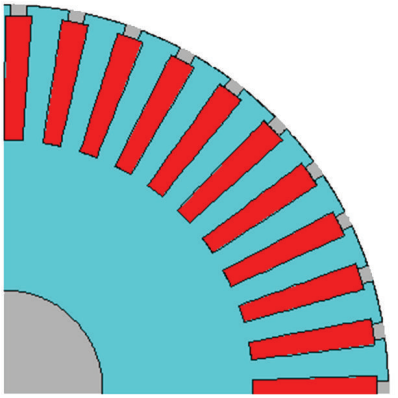

(e)

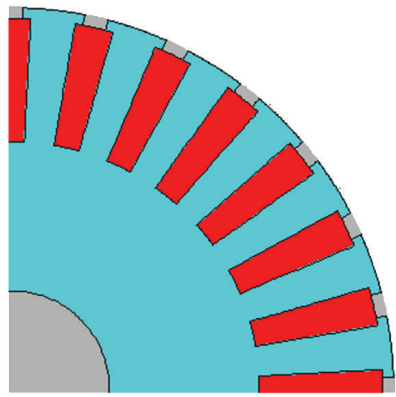

(b)

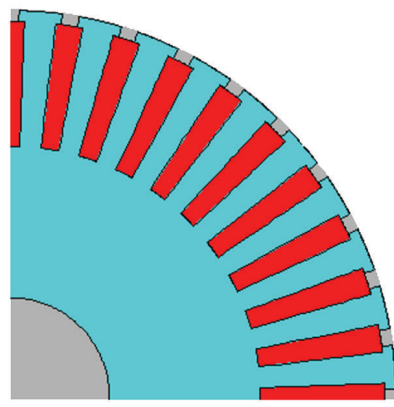

(d)

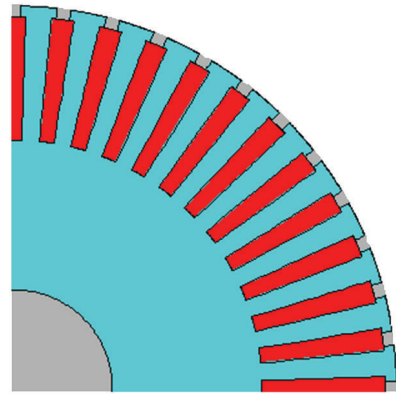

(f)
FIgURE 2: The simulated rotors with: (a) 24, (b) 28, (c) 30, (d) 40, (e) 41 , and (f) 48 rotor slots. 


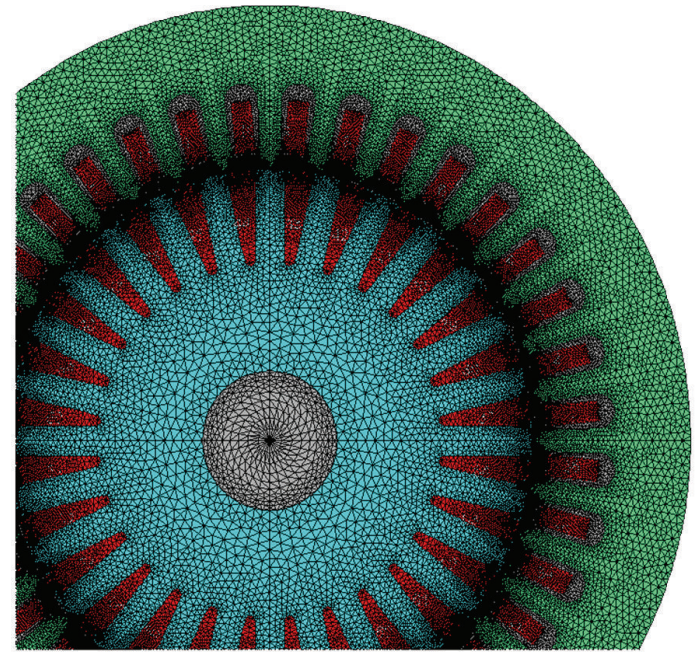

FIGURE 3: The mesh distribution in the motor with 28 rotor slots.
TABLE 1: The starting torque and starting current for all studied cases.

\begin{tabular}{lcc}
\hline Rotor slot number & Starting torque $(\mathrm{N} * \mathrm{~m})$ & Starting current $(\mathrm{A})$ \\
\hline 24 & 66.3 & 65.6 \\
28 & 73.3 & 67.2 \\
30 & 76.4 & 65.3 \\
40 & 72.4 & 65.6 \\
41 & 71.9 & 65.7 \\
48 & 74.4 & 65.7 \\
\hline
\end{tabular}

TABLE 2: The THD for every studied case.

\begin{tabular}{lc}
\hline Rotor slot number & THD (\%) \\
\hline 24 & 25.75 \\
28 & 22.64 \\
30 & 24.23 \\
40 & 18.4 \\
41 & 25.34 \\
48 & 19.03 \\
\hline
\end{tabular}

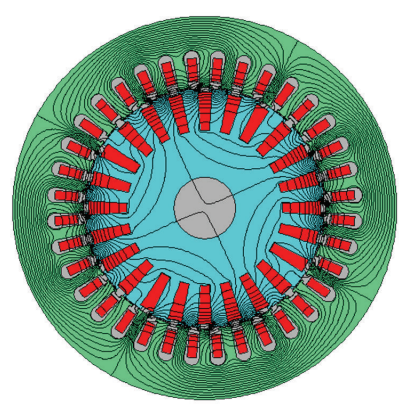

(a)

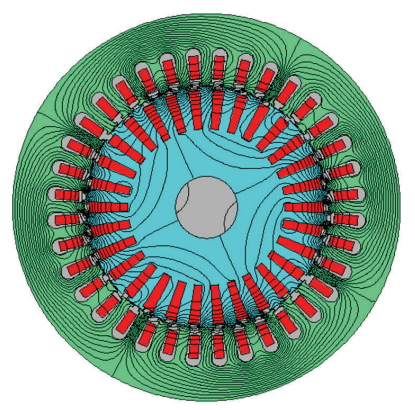

(c)

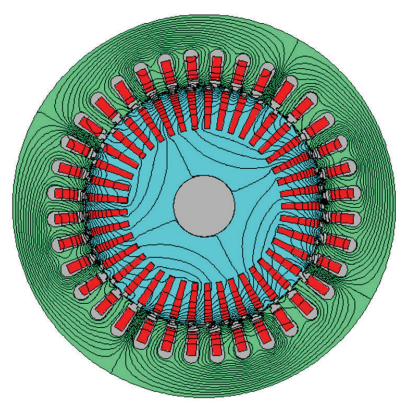

(e)

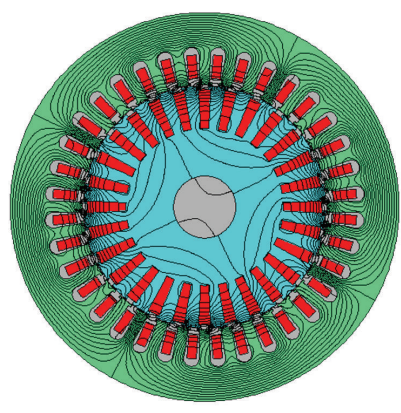

(b)

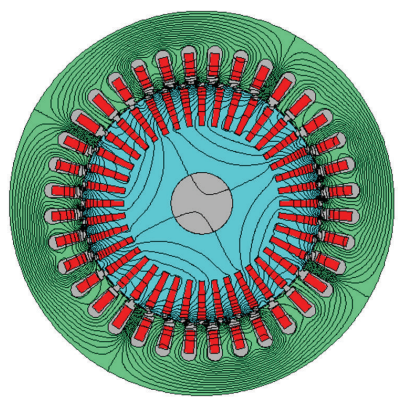

(d)

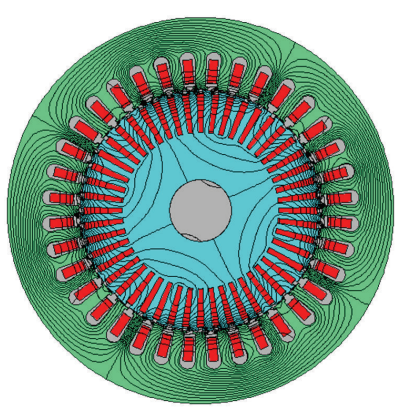

(f)
FIgURE 4: The magnetic flux lines distribution at starting for the motor with: (a) 24, (b) 28, (c) 30, (d) 40, (e) 41, and (f) 48 rotor slots. penetrate deep into the rotor core, as a consequence of the increased leakage flux. It is quite obvious that for the motor with 41 rotor slots, the magnetic flux lines penetrate the least into the rotor core, whereas in the motor with 24 slots they penetrate the most. Moreover, the increase of the rotor slot number has as an impact the stronger magnetization of the rotor parts close to the neutral zones of the magnetic field. If one compares the motor with 24 rotor slots (Figure $4(\mathrm{a})$ ) with the one having 48 rotor slots (Figure 4(f)), he will come to the conclusion that in the later the magnetic flux lines do not penetrate as deep into the rotor core, as the first one. On the other hand, the motor with 48 rotor slots presents greater magnetic flux density on the surface of the rotor core opposite to the neutral zones of the magnetic field, compared to the motor with 24 rotor slots.

In order to sufficiently compare the starting behavior of the motors, the starting torque and stator current were extracted for each case and they are presented in Table 1 . The motor with 24 rotor slots is characterized by the least starting torque, whereas the motor with 30 rotor slots has the greatest. The model with 30 rotor slots is also characterized by the least starting stator current amplitude. The motor with 28 rotor slots draws the greatest starting current. It is also remarkable that the motor with 48 rotor slots has the second greater starting torque for practically the same stator current with the other motors except the one with 28 rotor slots.

\section{Behavior at Nominal Speed}

5.1. Air Gap Space Harmonics. In this paragraph, the authors will show the air gap harmonic content for every case at $1440 \mathrm{rpm}$. The spatial harmonic content is the result of the stator and rotor MMF harmonics index, the slot existence, and the saturation of the iron cores. The magnitude of the air gap magnetic flux density is presented for every case in $\mathrm{dB}$ in Figure 5. 


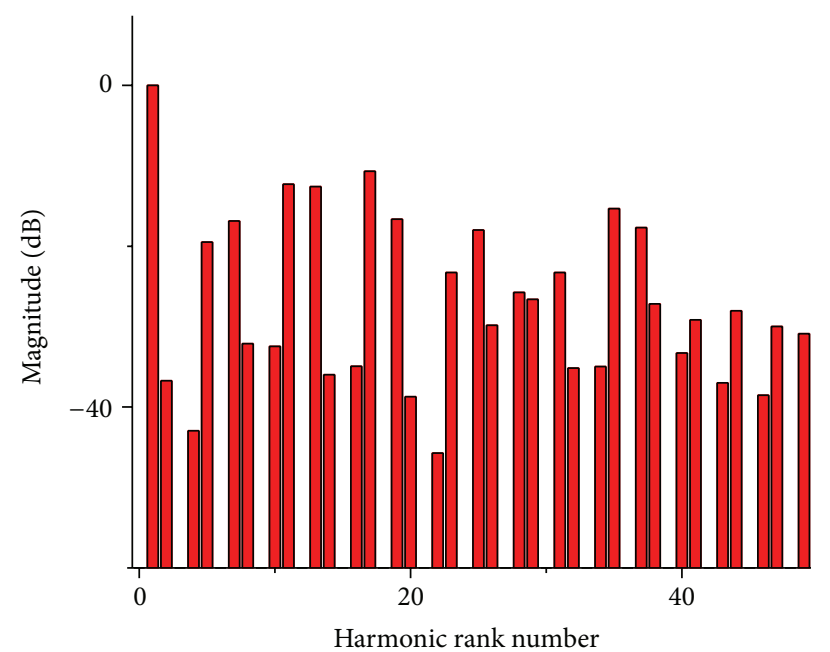

(a)

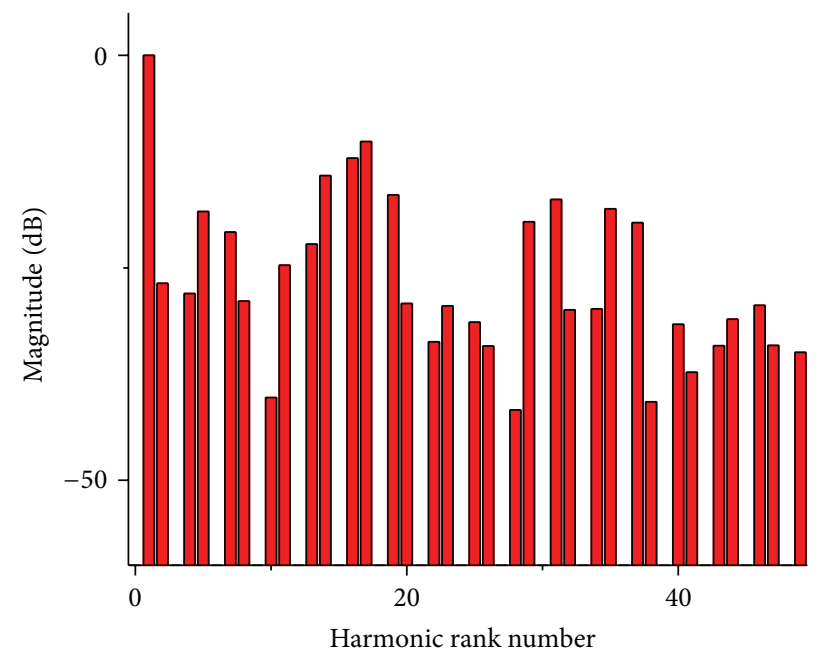

(c)

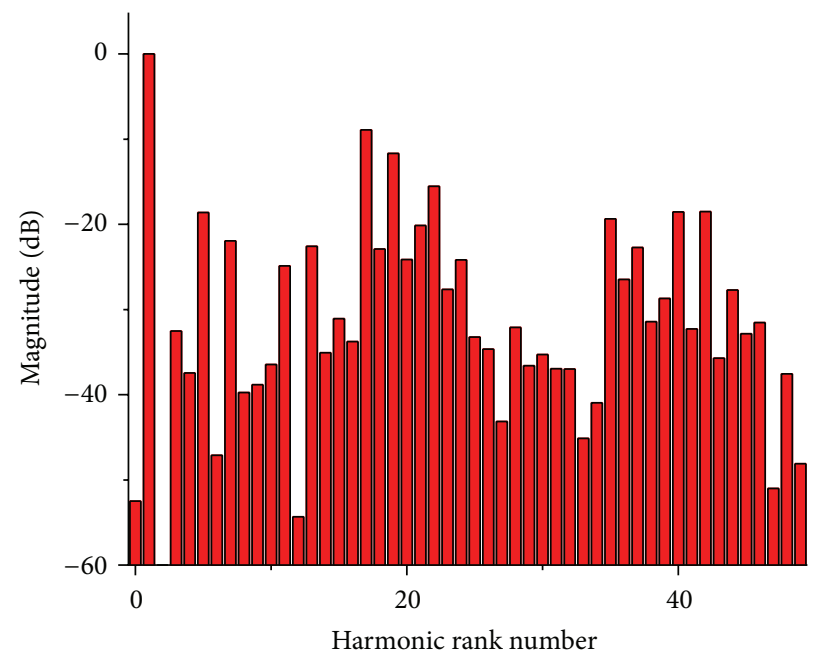

(e)

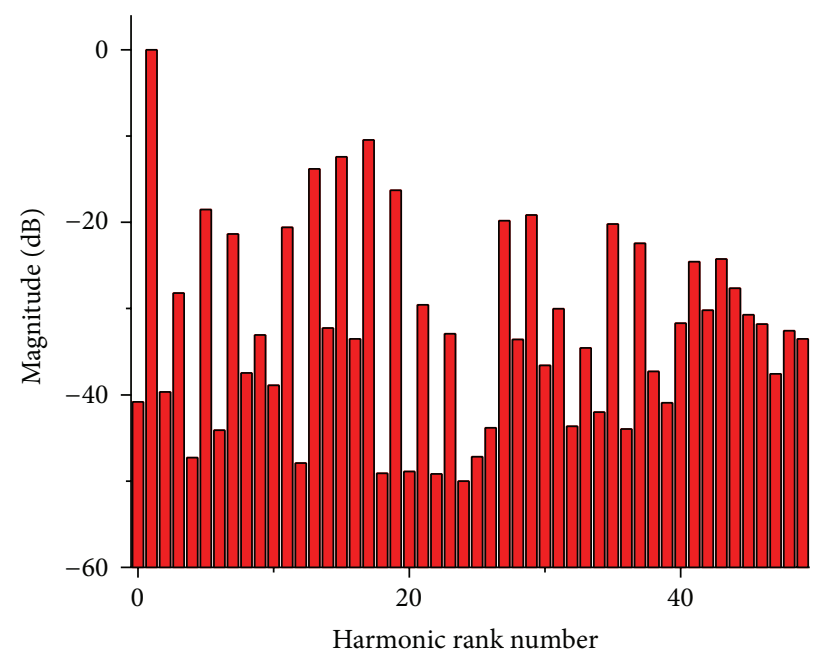

(b)

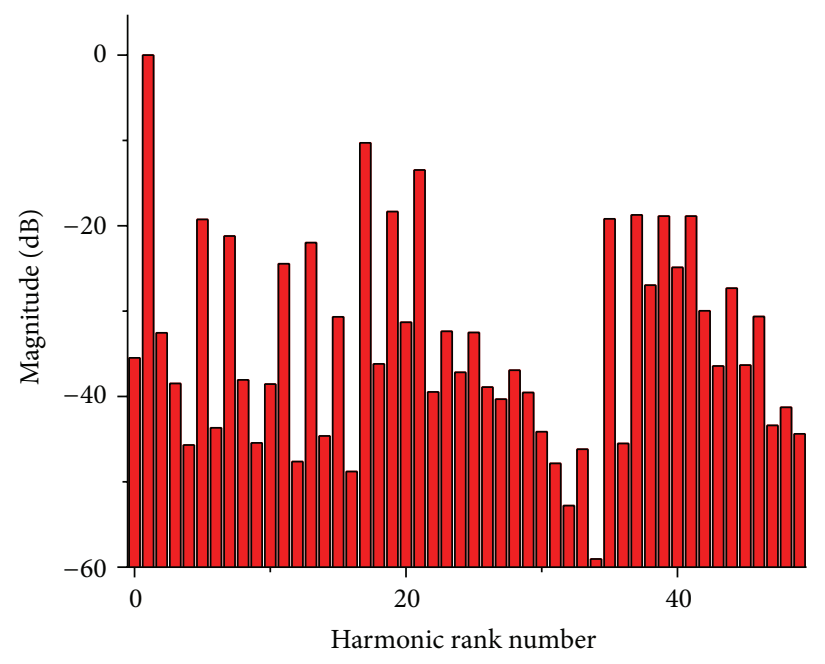

(d)

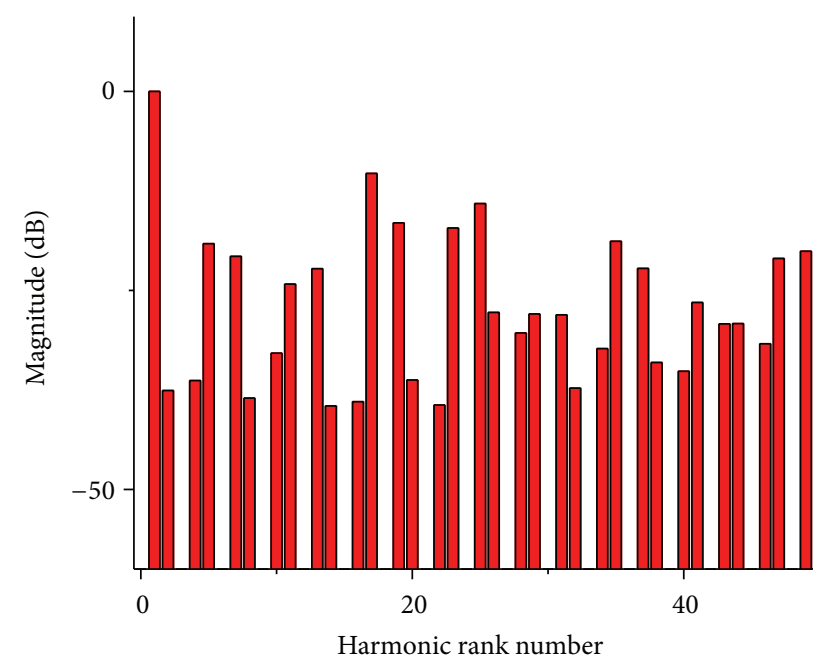

(f)

Figure 5: The magnitude of the air gap magnetic flux density at $1440 \mathrm{rpm}$ for the motor with: (a) 24, (b) 28, (c) 30, (d) 40, (e) 41 , and (f) 48 rotor slots. 


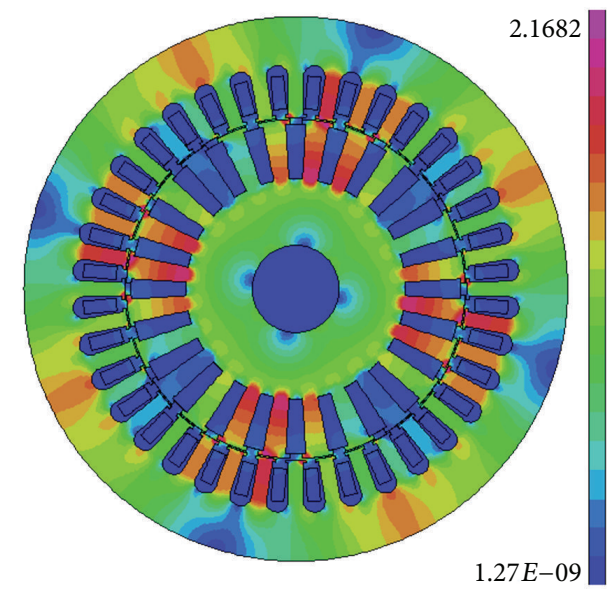

(a)

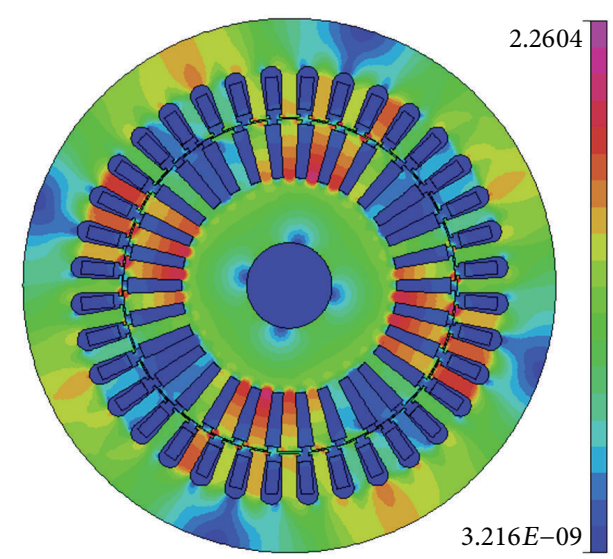

(c)

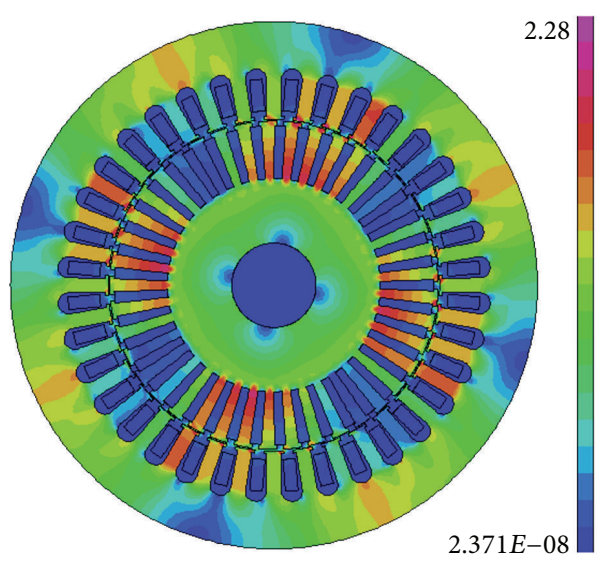

(e)

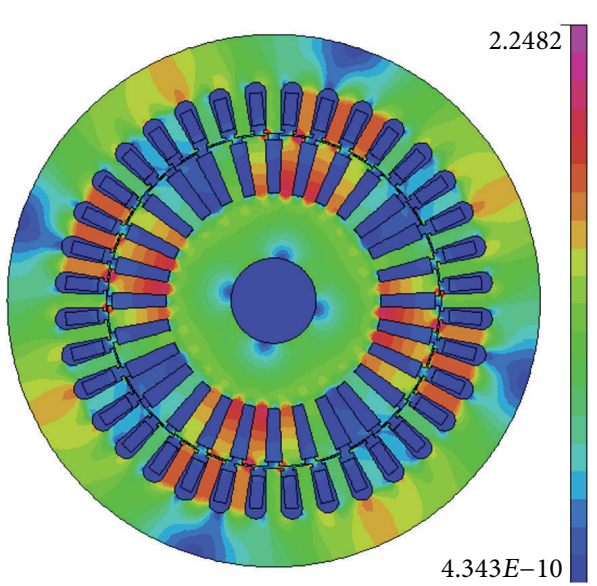

(b)

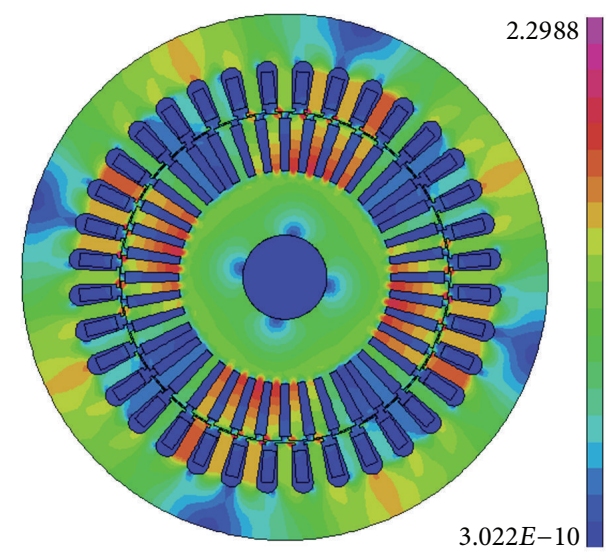

(d)

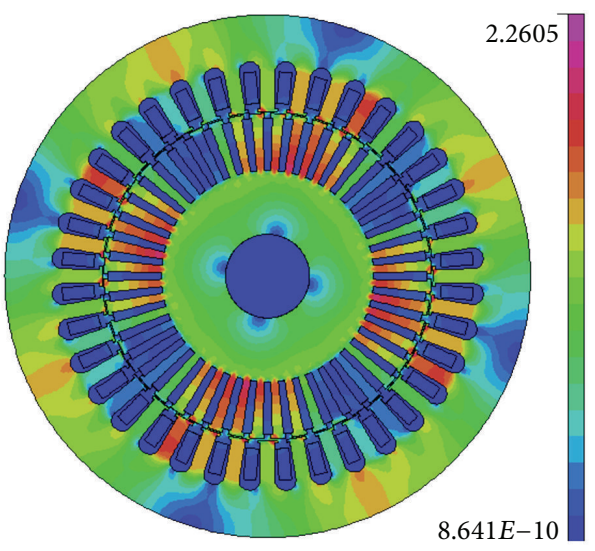

(f)

Figure 6: The spatial distribution of the amplitude of the magnetic flux density for the motor with: (a) 24, (b) 28, (c) 30, (d) 40, (e) 41, and (f) 48 rotor slots. 
TABLE 3: Characteristics of the motors at $1440 \mathrm{rpm}$.

\begin{tabular}{|c|c|c|c|c|c|c|}
\hline Rotor slots & Torque $(\mathrm{N} * \mathrm{~m})$ & Current (A) & $\operatorname{Cos} \varphi$ & Pin $(W)$ & Pout $(W)$ & $\eta(\%)$ \\
\hline 24 & 44.93 & 12.79 & 0.85 & 8764 & 6772 & 77.3 \\
\hline 28 & 48.2 & 12.61 & 0.81 & 8201 & 7264 & 88.6 \\
\hline 30 & 48.52 & 12.61 & 0.85 & 8693 & 7313 & 84.1 \\
\hline 40 & 49.09 & 12.55 & 0.87 & 8770 & 7399 & 84.3 \\
\hline 41 & 48.95 & 12.53 & 0.86 & 8713 & 7378 & 84.7 \\
\hline 48 & 50.73 & 12.49 & 0.86 & 8704 & 7646 & 87.8 \\
\hline
\end{tabular}

Firstly, it is obvious that there is a resemblance between Figures 5(a), 5(c), and 5(f). In those cases, the multiples of 3 harmonics are absent. This happens because the rotor slots in those cases are 24, 30, and 48, numbers which are multiples of 3. In all motors, the stator MMF and stator-slotrelated harmonics (such as 5, 7,11, and 13) are characterized by similar amplitudes. In order to have a better overview of the higher harmonic content, in every case the (total harmonic distortion) THD was calculated as an amplitude ratio. The results are presented in Table 2.

From Table 2, one can see that the worst cases are those with 24 and 41 rotor slots. In the case of the motor with 24 rotor slots, the result is easily explicable. The low rotor slot number has the impact of more and stronger rotor-slotrelated harmonics (such as 23 and 25).

The motor with 41 rotor slots is obviously a motor with odd rotor slot number. As a consequence, the even higher harmonics have increased amplitudes. This agrees with ignore. Previous work state that motors manufactured with odd rotor slot number are very noisy and have increased mechanical oscillations [18].

Best cases seem to be the motors with 40 and 48 rotor slots. Their rotors have many slots, and as a consequence their rotor-slot-related harmonics are met in higher ranks and so they have less impact. Also, both numbers are multiples of 4, a fact that has strong impact on the reduction of the rotor and stator-MMF-related higher harmonics, since the motors have four magnetic poles, as already mentioned in Paragraph 2.

5.2. Characteristics at $1440 \mathrm{rpm}$. In this paragraph, the characteristics of the six motors are presented for speed $1440 \mathrm{rpm}$. This speed is chosen as a typical value for motors of this size and characteristics. The calculated results are shown in Table 3. The motor with 24 rotor slots has the worst behavior since it presents the least output power together with the least efficiency, and at the same time draws the highest current. Generally, one can claim that for the motors with even rotor slot number, while the slot number increases, their electromagnetic torque and as a consequence their output power, increase also.

Moreover, the motor with 28 rotor slots is characterized by the highest efficiency but on the other hand by a poor power factor.

Furthermore, the motor with 41 rotor slots has an acceptable behavior, since its characteristics are literally an average as compared to the others.

The motor with 40 rotor slots is characterized by the greatest power factor, but by lower efficiency than the motors with 28 and 48 rotor slots.
Finally, the motor with 48 rotor slots presents the greatest electromagnetic torque and output power. At the same time, this motor draws the least stator current. The power factor and the efficiency are the second best. If one takes into consideration the results from the previous paragraphs, it is reasonable to claim that for the stator used, the rotor slot number 48 offers the best motor behavior in general, which means good starting characteristics, high efficiency and power factor, low stator current, and less vibrations and mechanical oscillations at nominal speed.

5.3. Spatial Distribution of the Magnetic Flux Density. In order to gain insight of the numerical results presented in Sections 5.1 and 5.2, the magnetic flux density amplitude has been charted for all models and the results are presented in Figure 6.

It is clear that the motor with 40 rotor slots presents the greatest amplitude of the magnetic flux density, where the motor with 24 rotor slots presents the least. Moreover, the motor with 24 rotor slots seems to be more saturated and especially at the tooth tips. Furthermore, in the motors whose numbers are not a multiple of 4 , such as in Figures 6(c) and 6(e), it is clear that there exists an asymmetry on the stator teeth magnetic flux density distribution. This is the reason why these motors, with 30 and 41 rotor slots, were characterized by increased THD in Table 2 .

\section{Conclusions}

This work studies the influence of several rotor slot numbers on the behavior of the induction motor. FEM analysis indicates that for the selected number of stator slots and number of magnetic poles, the rotors with slot number multiple of 3 , do not have higher harmonics multiples of 3 in the air gap magnetic flux density. Also, the extracted results lead to the conclusion that the motor with 48 rotor slots is the best case, while it presents good starting behavior along with low air gap magnetic flux density harmonic content and improved characteristics at nominal speed.

\section{Nomenclature}

a: $\quad$ Geometrical angle

$\lambda_{g 1}, \lambda_{g 2}$ : Component of the air gap relative specific permeance varying with the angle $\alpha$ when only the stator and rotor, respectively, are slotted

$\tau: \quad$ Stator slot pitch

$\omega: \quad$ Stator angular frequency

$\omega_{2}$ : Rotor angular frequency 


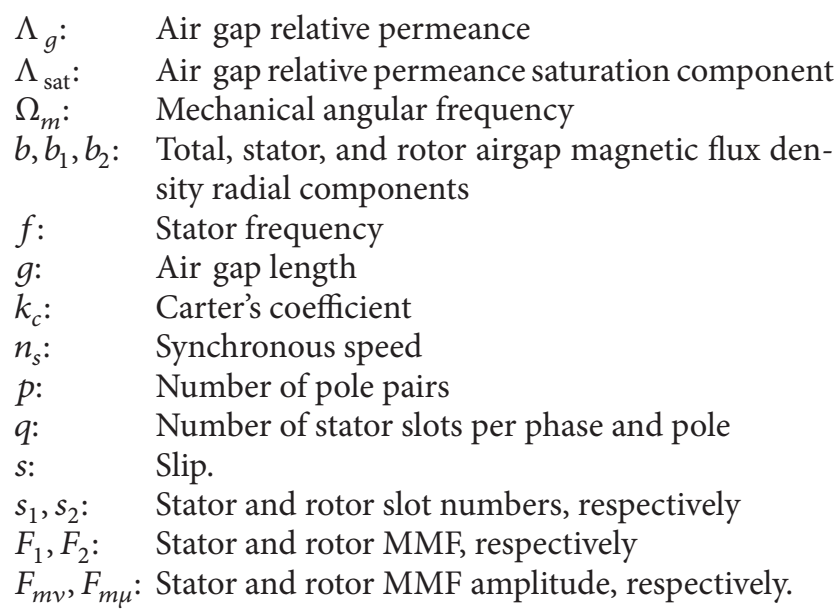

\section{Acknowledgment}

This work has been supported by the Research Program "K. Karatheodori 2010" of the Research Committee of the University of Patras, Greece.

\section{References}

[1] T. J. McCoy and J. V. Amy Jr., "The state-of-the-art of integrated electric power and propulsion systems and technologies on ships," in Proceedings of the IEEE Electric Ship Technologies Symposium (ESTS '09), pp. 340-344, April 2009.

[2] J. M. Prousalidis and P. S. Mouzakis, "Analysis of electric power demands of podded propulsors," Journal of Marine Engineering and Technology, vol. 2010, no. 16, pp. 3-13, 2010.

[3] G. D. Marques and D. M. Sousa, "A new sensorless MRAS based on active power calculations for rotor position estimation of a DFIG," Advances in Power Electronics, vol. 2011, Article ID 970364, 8 pages, 2011.

[4] L. Charaabi, "FPGA-based fixed point implementation of a realtime induction motor emulator," Advances in Power Electronics, vol. 2012, Article ID 409671, 10 pages, 2012.

[5] J. Pyrhonen, T. Jokinen, and V. Hrabovcova, Design of Rotating Electrical Machines, John Wiley \& Sons, 1st edition, 2008.

[6] S. Nandi, "Modeling of induction machines including stator and rotor slot effects," IEEE Transactions on Industry Applications, vol. 40, no. 4, pp. 1058-1065, 2004.

[7] J.-W. Kim, B.-T. Kim, and B. I. Kwon, “Optimal stator slot design of inverter-fed induction motor in consideration of harmonic losses," IEEE Transactions on Magnetics, vol. 41, no. 5, pp. 20122015, 2005.

[8] J. Kappatou, K. Gyftakis, and A. Safacas, "FEM study of the rotor slot design influences on the induction machine characteristics," in Studies in Applied Electromagnetics and Mechanics, vol. 30 of Advanced Computer Techniques in Applied Electromagnetics, IOS Press, 2008.

[9] I. Boldea and S. A. Nasar, The Induction Machines Design Handbook, Taylor \& Francis Group, 2010.

[10] J. F. Gierras, C. Wang, and J. C. Lai, Noise of Polyphase Electric Motors, CRC Press, 2006.

[11] M. Dabrowski, Magnetic Circuits and Fields of Electrical Machines, WNT, Warsaw, Poland, 1971.

[12] A. Voldek, Electrical Machines (in Russian), Energia, Leningrad, Russia, 2nd edition, 1974.
[13] B. Heller and V. Hamata, Harmonic Field Effects in Induction Machines, Academia Publishing House, Prague, Czech Republic, 1977.

[14] W. Weber, Der Nutungsfaktor in Elektrischen Maschinen, ETZ, 1928.

[15] H. Frohne, Über die Primären Bestimmungsgrößen der Lautstärke bei Asynchronmaschinen [Ph.D. thesis], Hannover, Germany, 1959.

[16] M. Purkemani, Beitrag zur Erfassung der Sattigungsoberfelder in Drehstromasynchromaschinen [Ph.D. thesis], Technischen Hochschule Hannover, Fakult at fur Maschinenwesen, Hannover, Germany, 1971.

[17] J. Le Besnerais, V. Lanfranchi, M. Hecquet, and P. Brochet, "Optimal slot numbers for magnetic noise reduction in variable-speed induction motors," IEEE Transactions on Magnetics, vol. 45, no. 8, pp. 3131-3136, 2009.

[18] I. Boldea and S. A. Nasar, The Induction Machines Design Handbook, CRC Press, 2nd edition, 2010. 

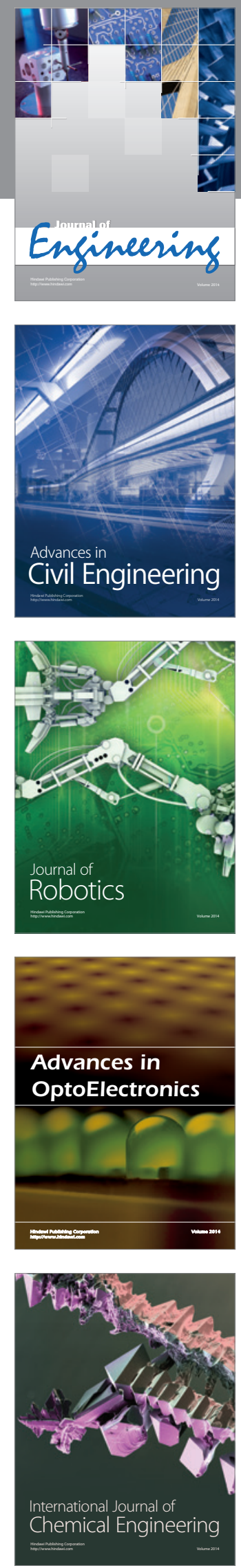

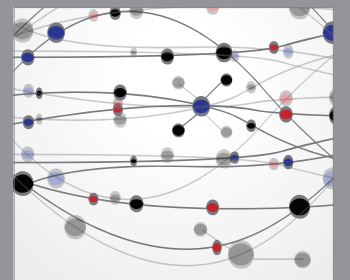

The Scientific World Journal
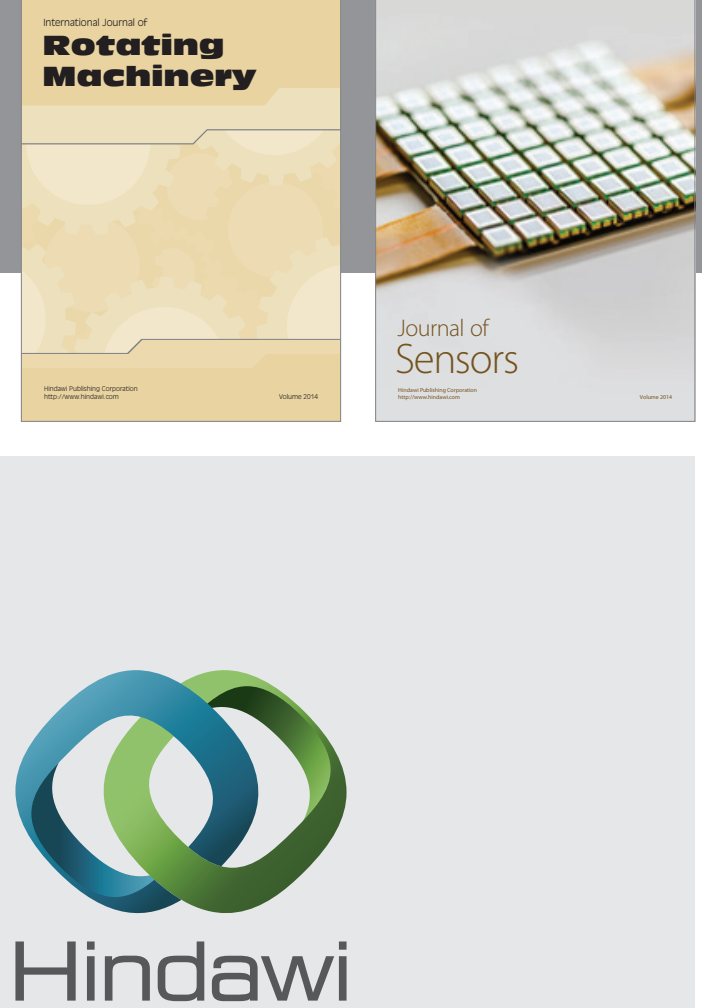

Submit your manuscripts at http://www.hindawi.com
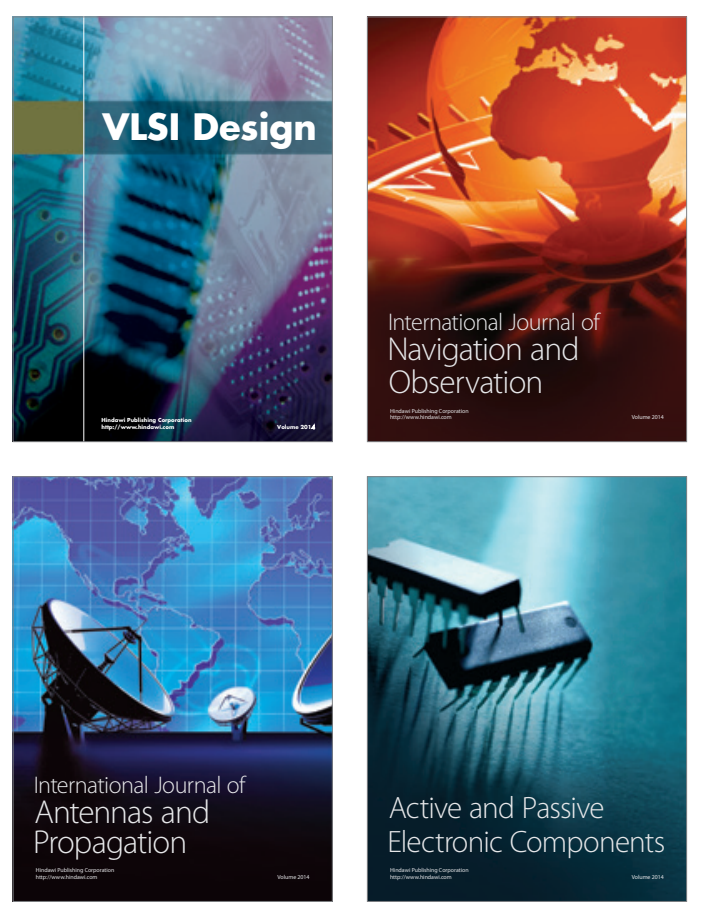
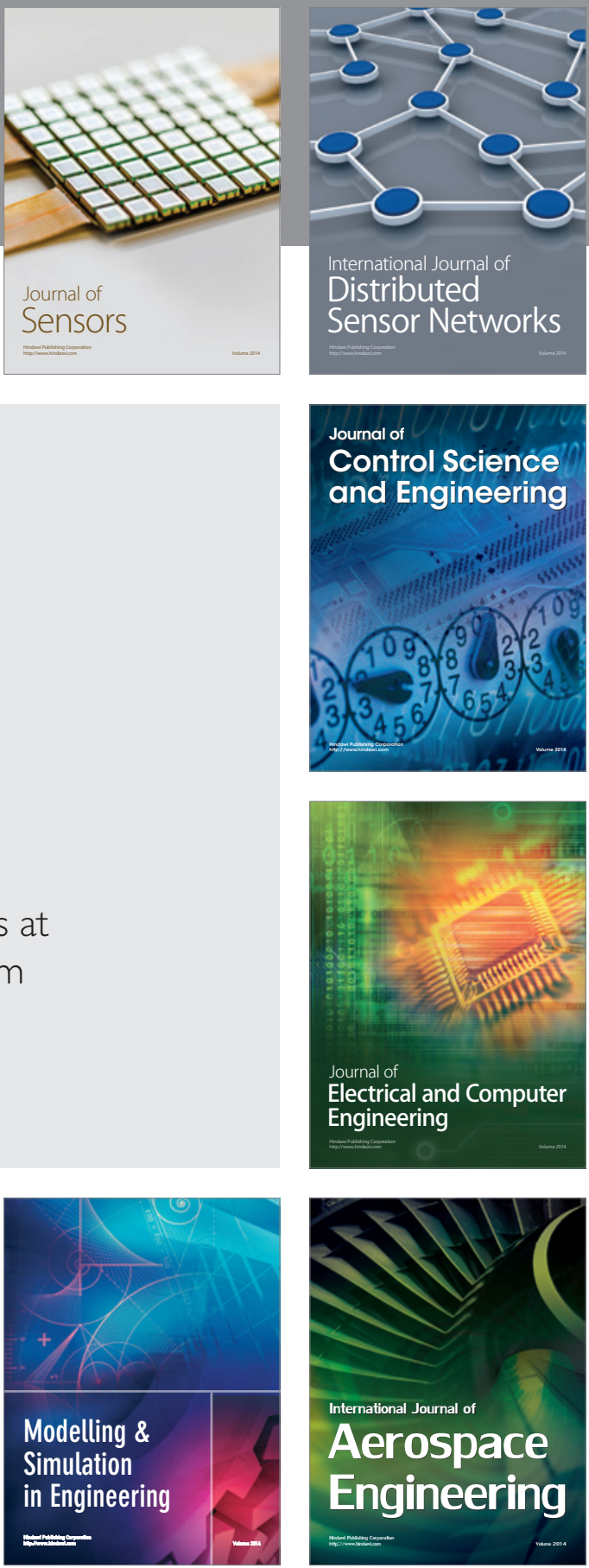

Journal of

Control Science

and Engineering
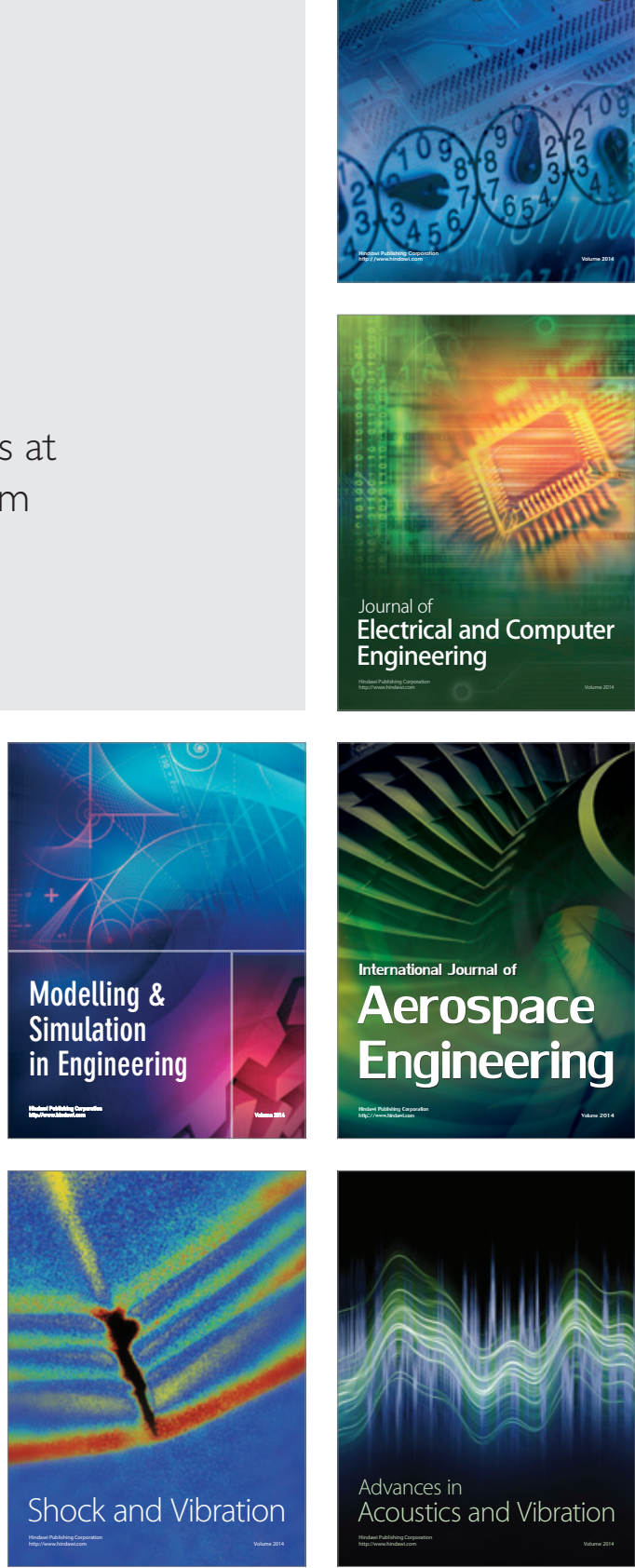